\title{
Die Blechtrommel und die italienische Kulturszene Anfang der Sechziger Jahre
}

\author{
Eva Banchelli
}

\begin{abstract}
This contribution discusses the historical and cultural context in which the first Italian translation of Die Blechtrommel was published (1962). The relatively late date of publication was due to the political and religious conservatism of the country. Even the left wing cultural scene was marked by a certain dogmatic conformism. This explains the scruples with which Italian publishers read and then rejected Die Blechtrommel. The literary climate only began to change in the beginning of the 1960s with the appearance of journals like Il Verri. The aesthetic programme of the authors and critics who now started to publish had strongly resonated with the transition that was going on in Germany in the same period. The innovations soon had strong repercussions in the publishing sector, especially with regard to the import of foreign works. The change in fortunes for Grass's novel came when the Feltrinelli publishing house, founded in Milan in 1955, started to take a serious interest in new experimental German literature. This was largely thanks to the enthusiasm and commitment of the young editor Enrico Filippini. The Italian translation finally appeared in the renowned series „I narratori“, which also included other esteemed names from contemporary literature. The novel was presented to the public as a radical representative of experimental iconoclastic literature, an image that was confirmed in the controversial critical reception accorded to the novel.
\end{abstract}

In den Sechziger Jahren war die Frage:

„Was halten Sie von Grass?" so häufig,

dass sie im Deutschen der von

„Aimez-vous Brahms?“ gleichkam. ${ }^{1}$

1 Sorge (2003).

(C) EVA BANCHELLI, 2016 | DOI 10.1163/9789004291898_006

This is an open access chapter distributed under the terms of the CC-BY-NC License. 
Die italienische Übersetzung von Die Blechtrommel ${ }^{2}$ erschien im Oktober 1962 in Italien nach Überwindung einer Woge des Misstrauens und der Verrisse seitens der Verlage. Das im Vergleich zur deutschen Originalausgabe von 1959 und den wichtigsten Sprachen im Westen (französische Übersetzung 1960; englische Übersetzung 1961) verspätete Erscheinungsdatum war nur am Rande durch die Problematik bedingt, einen für diese schwierige Aufgabe geeigneten Übersetzer ausfindig zu machen. In Wirklichkeit stellte vor allem das politische und kulturelle Klima gegen Ende der 5oer Jahre ein Hindernis für die Bereitschaft zur Rezeption und Verbreitung des Romans von Grass dar.

Das Land durchlebte damals eine Phase der tiefgreifenden Umwandlung und Neuorientierung, geprägt von einschneidenden Veränderungen, aber auch von einem starken Ungleichgewicht und von einer Kontinuität in Bezug auf die Vergangenheit. Erst ab dem Sommer 196o begann man mit der Amtsübernahme der neuen Regierung unter dem Vorsitz von Amintore Fanfani und mit einer ersten Öffnung gegenüber der sozialistischen Partei allmählich aus der Nachkriegszeit mit ihrem kompakten klerikalen Moderatismus herauszufinden, welcher während der kurzen christdemokratischen Regierung unter Fernando Tambroni (von März bis Juli 1960) einen extremen Ausbruch von Autorität und Repression erfahren hatte. Die politische Wende hin zu einer Mitte-Links-Koalition, die mit der Bejahung des Neo-Kapitalismus und der Verbreitung eines Wirtschaftswunders einherging, wurde durch den Einfluss der kirchlichen Macht des Vatikans beeinträchtigt, der, ungeachtet der von Papst Roncalli unternommenen Bemühungen hinsichtlich einer Erneuerung (ab 1959), weiterhin beträchtlichen Einfluss auf die gemeinsamen Moralvorstellungen und die kulturelle Ausrichtung der breiten Öffentlichkeit nahm. Unverändert blieb beispielsweise die Unnachgiebigkeit der Kirche angesichts der heiligen Unantastbarkeit des ehelichen Bundes, und die 1949 vom Heiligen Stuhl beschlossene Exkommunikation derer, die sich zur kommunistischen Lehre bekannten, sie verteidigten und propagierten, wurde aufrechterhalten.

Das allgemeine Klima wurde folglich vom ständigen Kompromiss zwischen laizistischen und fortschrittlichen Ansprüchen und einem alten, eingesessenen und mächtigen Konservatismus katholischer Prägung beeinflusst. Obgleich Artikel 21 der Verfassung die Freiheit der Presse und jeglicher anderer Ausdrucksformen gewährte, nahm es nicht Wunder, dass ein Gesetz zur Anwendung kam, das am 31. Mai 1946 vom damaligen christdemokratischen Premierminister De Gasperi und dem kommunistischen Justizminister

2 Günter Grass, Il tamburo di latta. Übersetzt von Alfredo Fölkel und Lia Secci (Milano: Feltrinelli, I narratori, 1962). 
Palmiro Togliatti unterzeichnet wurde und die Beschlagnahmung von als obszön geltenden oder gegen die allgemeine Sittlichkeit verstoßenden Publikationen vorsah. Gegen Ende der 5oer Jahre schnellten die Fälle von Beschlagnahmungen in der Publizistik und Literatur, die man der Verletzung des Schamgefühls und Beleidigung der Religion bezichtigte, geradezu in die Höhe. ${ }^{3}$

Ein Roman, wie der von Grass, der auch in der konservativen BRD Adenauers wohlbekannte Skandale heraufbeschworen hatte, war also in Italien in erster Linie einem hohen Risiko der Zensur und den moralischen Bedenken selbst angesehener und aufgeklärter Verlage ausgesetzt. Dies war nämlich der Fall beim ersten Verleger, der sich an der Blechtrommel versuchte, dem Mailänder Valentino Bompiani. Durch eine italienische Rezension mit der Unterschrift eines Paolo Milano in der Wochenzeitschrift L'Espresso auf den Erstling von Grass aufmerksam geworden, ${ }^{4}$ erwarb dieser sofort die Rechte am Roman. Jedoch verzichtete er im Mai 1962 mit bereits 5000 gedruckten Exemplaren darauf, das Buch auf den Markt zu bringen und ließ es einstampfen, nachdem er mit einiger Verzögerung die Übersetzung gelesen hatte.

Wie aus den Erinnerungen von Enrico Filippini hervorgeht - auf den nachfolgend noch eingegangen wird - nahm Bompiani, ein intelligenter und kosmopolitischer Mensch, allerdings ein Gegner jeglicher Art der literarischen Provokation, Anstoß „vor allem [an] der Szene, in der der Zwerg Matzerath entschlossen seine Zähne im Schamhaar eines Kindermädchens versenkt". ${ }^{5}$ In jüngst erschienenen Forschungen ${ }^{6}$ wird indessen behauptet, vor allem die blasphemischen Stellen im Roman, insbesondere im Kapitel „Kein Wunder“, hätten Bompiani zu dieser Reaktion veranlasst, der schließlich auch nach Anhörung seiner engsten Mitarbeiter sämtliche Rechte und die Übersetzung an Feltrinelli abgetreten habe.

Um den darauf folgenden holprigen Weg des Romans in Italien bis zu seinem glücklichen Ende, der Publikation, nachvollziehen zu können, sei nun der Blick auf die linke Kulturszene gerichtet, die wiederum von starken Spannungen und miteinander kontrastierenden Strömungen geprägt war. Nach Chrustschtows Enthüllungen über Stalins Verbrechen und die Geschehnisse in Ungarn war die solide politische und kulturelle Hegemonie der PCI zerbrochen. Viele,

3 Vgl. Armano (2013).

4 Milano (1960) stellte den Roman als ein Novum in der deutschen Literatur vor und bemerkte, dass Grass trotz der offenkundigen Schwächen und Exzesse seines Textes der Meister einer zügellosen Fabulierlust gewesen sei.

5 Filippini (1978). Übersetzungen der italienischen Zitate von E. B.

6 Di Stefano (2007); Ferretti (2012) 117-118. 
vor allem aus den Reihen der Intellektuellen, lehnten deren Treue zur UdSSR und den ideologischen Dogmatismus ab. Am 29. Oktober 1956 wurde das Manifesto dei 101 veröffentlicht, worin aus der kommunistischen Intelligenzia Italiens hervorstechende Persönlichkeiten ebenfalls ganz offen gegen die Mauer der Orthodoxie in ihrer Partei ankämpften. Ein Jahr später, nachdem der achte Kongress der PCI die Hoffnungen auf eine Wende der Erneuerung enttäuscht hatte, trat der Schriftsteller Italo Calvino mit einem offenen Brief an die Tageszeitung L'Unità aus der Partei aus. Calvino ist besonders in dem hier geschilderten Zusammenhang interessant, denn er war schon lange eine herausragende Persönlichkeit beim Verlagshaus Einaudi, welches sich, wie wir sehen werden, kurz darauf seinerseits mit der Entscheidung für oder gegen eine Veröffentlichung Der Blechtrommel auseinandersetzte. Der als sehr junger Partisan der kommunistischen Partei beigetretene Schriftsteller begründete seinen Austritt nicht nur im Namen eines politischen Dissenses, sondern auch damit, dass er die in der kulturellen und ästhetischen Ausrichtung der Partei wahrgenommenen Verschlossenheit nicht ertragen könne:

Ich habe nie gedacht (auch nicht im anfänglichen Eifer des Neulings), dass die Literatur jene traurige Angelegenheit sei, die viele in der Partei predigen, und gerade die Armseligkeit der offiziellen Literatur des Kommunismus diente mir bei dem Versuch meine schriftstellerische Arbeit unter das Zeichen schöpferischen Glücks zu stellen als Ansporn. ${ }^{7}$

Bei seiner Gegenüberstellung von traurigem Konformismus und schöpferischer Kreativität brachte Calvino ein Unbehagen mit unterschiedlichen Auswirkungen auf das literarische Feld zum Ausdruck, das im ersten Jahrzehnt der Nachkriegszeit vom Paradigma des Neo-Realismus beherrscht war. Als Autor von I sentieri dei nidi di ragno (Wo Spinnen ihre Neste bauen, 1947), einem der repräsentativsten Romane dieser literarischen Strömung, bekam Calvino nunmehr, wie auch viele seiner Kollegen, deren formale wie inhaltliche Grenzen zu spüren.

Die neo-realistische Ästhetik - mit ihrem moralistischen Populismus, ihrer ideologischen Reduzierung der Komplexität der Wirklichkeit und ihren tapferen Plädoyers für das literarische Engagement - blieb dennoch eines der unangetastetsten Dogmen der italienischen linken Kultur. Diese war Lukacs' Konzept der Widerspiegelung und dem von Sartre und Brecht propagierten Modell des Intellektuellen stark verhaftet.

7 Calvino (1957). 
Nachdem sich der ideale Antrieb des Widerstandes und der Hoffnung auf eine radikale Veränderung der politischen Realität des Landes erschöpft hatte, brachte dagegen nun eine Gruppe zahlreicher Intellektueller und Künstler die eigene Unzufriedenheit mit Entscheidungen auf dem Gebiet der Erzählliteratur und Sprache zum Ausdruck, die ihnen angesichts der schwierigen Zeit und der Bedrohungen seitens des sich ausbreitenden Konformismus unangemessen schienen. ${ }^{8}$ Eines ihrer wichtigsten Sprachrohre war die 1956 gegründete Zeitschrift Il Verri, unter der Leitung des bekannten Luciano Anceschi, Professor der Ästhetik an der Universität Bologna. Die Personen im Umfeld dieser Zeitschrift - Renato Barilli und Angelo Guglielmi, Elio Pagliarani und Nanni Balestrini, um nur einige zu nennen - verstanden sich als ,Neuavantgardisten' und sollten kurz danach die Gruppe 63 bilden, die sich ganz offen als Schriftsteller- und Kritikerverband am Modell der Gruppe 47 jenseits der Alpen orientierte. Allerdings hatte diese neue ästhetische Richtung viele Berührungspunkte mit der Veränderung, die auch die deutsche Literatur in derselben Zeit erfuhr; ein Anzeichen für ein gesellschaftspolitisches Klima, das wiederum nicht wenige Gemeinsamkeiten mit dem in Italien hatte. Es soll genügen, auf die Bedeutung des ,mythischen' Jahres 1959 einzugehen, in dessen Zusammenhang neueste Studien auf „die intellektuelle Gründung der Bundesrepublik" verweisen. ${ }^{9}$ In diesem Jahr hatten bekanntlich die Vertreter einer neuen Schriftstellergeneration ihren triumphalen Auftritt. Nicht nur die immer wieder erwähnten Autoren wie Martin Walser, Uwe Johnson und Grass selbst veröffentlichten ihre revolutionären Romane, sondern auch Hans Magnus Enzensberger begann seine Karriere als Literaturkritiker, und der Älteste, Günther Eich, hielt anlässlich der Büchner Preisverleihung eine Rede zur poetischen Sprache, „als Gegnerschaft und Widerstand ${ }^{“ 10}$, die Geschichte machen sollte. ${ }^{11}$

Selbstverständlich betrachteten die Neuavangardisten die deutschen Kollegen mit großem Interesse, sobald sie damit beschäftigt waren, das Blatt in der italienischen Erzählliteratur zu wenden. Die Offensive gegen die verschiedenen Ausprägungen des vorherrschenden Realismus ging von Zeitschriften wie Il Verri, Quindici, Malebolge aus, mittels einer hervorragenden Gruppe von Literaturkritikern, denen Autoren wie Pratolini, Cassola, Bassani, selbst Pasolini mit Ragazzi di vita wie naturalistische Epigonen und konventionelle Diener des Gemeinsinns im Namen eines moralistischen und kurzsichtigen

\footnotetext{
8 Vgl. Il Verri, 1, 1960 [Sonderheft: La nuova narrativa italiana fra il 1945 e il 196o].

9 Albrecht (2000).

10 Abgedruckt in Büchner-Preis-Reden 1951-1971 (Stuttgart: Philipp Reclam jun., 1972), 86.

11 Berbig (2008); Häntzschel (2009).
} 
Engagements vorkamen. ${ }^{12}$ Im In- und Ausland gaben nun andere Modelle den Ton an: die klassische Moderne von Svevo, Joyce, Proust, Kafka, Döblin, Woolf, und unter den zeitgenössischen Werken vor allem der Nouveau Roman in Frankreich und in Italien der Meister Carlo Emilio Gadda, der durch die sprachliche Spannung einer ideologiefeindlichen Realitätsnähe die höchste Intensität zum Ausdruck zu bringen vermochte. ${ }^{13}$

Die neuen Talente, die uns hier mit Einschränkung auf den Bereich der Erzählliteratur am meisten interessieren, heißen Alberto Arbasino, Edoardo Sanguineti und Giorgio Manganelli: Deren Verdienst - wie beispielsweise der Literaturkritiker Angelo Guglielmi 1959 bemerkt - habe darin bestanden , „an und für sich ohne Hintergedanken auf eine gepflegte oder irgendeine andere Art und Weise zu erzählen", ${ }^{14}$ unter völliger Entzauberung angesichts der Faszination der Wirklichkeit, „an deren Schwere und Stumpfsinnigkeit viele Talente zerbrochen sind, die das Unrecht begangen hatten, sich ihr seit der Nachkriegszeit bis heute ausgesetzt zu haben“. ${ }^{15}$ Die Aufmerksamkeit verlagert sich von den Inhalten und dem Tatsachenmaterial, auf denen das Erzählen basiert, und von der zu vermittelnden moralischen oder ideologischen Botschaft auf die Erforschung der Sprache und das Experimentieren mit neuen Erzähltechniken. Um wahrhaft kritisch und realistisch sein zu können - so resümiert später Edoardo Sanguineti recht eindrucksvoll - muss die Kunst mit aller Entschlossenheit die Grenzen der bürgerlichen Normalität überschreiten, und zwar ihre ideologischen und sprachlichen Normen. ${ }^{16}$

Die sich in der italienischen Gesellschaft in den Jahren des Wirtschaftswunders vollziehenden Veränderungen brachten auch die Frage nach einer neuen Kultur und eine Geschmacksveränderung beim Publikum und der Literaturkritik mit sich. Gerade auf dieser Ebene konnte in jenem Moment eine Begegnung mit den innovativen deutschen Autoren stattfinden, die Ende der 5oer Jahre wiederum auf unterschiedliche Weise mit neuen Ausdrucksmöglichkeiten experimentierten, nachdem die innovativen Ressourcen des von der Gruppe 47 in der ersten Nachkriegszeit propagierten Realismus erschöpft waren. ${ }^{17}$ Und Die Blechtrommel spielt mit der Radikalität ihrer ikono-

\footnotetext{
12 Guglielmi (1960).

13 Guglielmi (1958).

14 Guglielmi (1959).

15 Guglielmi (1959) 153.

16 Barilli (1976) 273 .

17 Banchelli (2013) 553-561.
} 
klastischen Satire, mit ihrer „wilde[n] Energie des Ausdrucks" ${ }^{18}$ bekanntlich eine wesentliche Rolle bei diesem Umwandlungsprozess.

Diese grob skizzierten Entwicklungstendenzen im Bereich der Literatur hatten auch erhebliche Auswirkungen auf den Kultursektor und die italienische Verlagsgeschichte, insbesondere was die Einfuhr ausländischer Literaturen angeht. Auch hier lässt sich ein interessanter Paradigmenwechsel feststellen, der die Rezeption der deutschen Literatur beträchtlich beeinflussen sollte. ${ }^{19}$ Diese wurde bis dahin in erster Linie von den beiden wichtigsten Verlagshäusern des Landes vertrieben: von Mondadori mit Sitz in Mailand und Einaudi mit Sitz in Turin. ${ }^{20}$ Ersteres führte große Autoren des zwanzigsten Jahrhunderts in seinem Katalog, wie Thomas Mann, Hermann Hesse, Franz Kafka, Erich Maria Remarque und unter den zeitgenössischen Heinrich Böll, Luise Rinser und Alfred Andersch. Beraterin für den deutschen Sprachbereich war Lavinia Mazzucchetti, (1889-1965), der seit den 3oer Jahren in der italienischen Verlagslandschaft die nachhaltigste Aktivität der Kulturmediation mit Deutschland zu verdanken ist. Als persönliche Freundin von Thomas Mann war sie, wie aus den obengenannten Entscheidungen hervorgeht, eine überzeugte Verfechterin der klassischen Moderne und des ,kritischen Realismus im Sinne von Lukács.

Auch Einaudi, der in jenen Jahren alle großen Autoren des italienischen Neo-Realismus unter der Leitung des hervorragenden Germanisten marxistischer Prägung Cesare Cases $(1920-2005)^{21}$, herausbrachte, förderte die große Tradition des bürgerlichen Realismus und die engagierte Literatur, wobei er indes gegenüber dem Experimentalismus der Avantgarden äußerste Vorsicht an den Tag legte. Cases brachte die literaturkritischen Schriften von Lukacs nach Italien und ließ nicht ohne gewisse Bedenken Brecht und mit großer Begeisterung Heinrich Mann, Hermann Broch und Robert Musil, Anna Seghers und Stefan Heym übersetzen. ${ }^{22}$

Dem Verlag Mondadori, wie auch Einaudi, war das Aufsehen, das Die Blechtrommel 1958 durch die Auszeichnung der Gruppe 47 und 1959 auf der Frankfurter Buchmesse erregt hatte, gewiss nicht entgangen. Die von beiden Verlagshäusern verfolgte moderate kulturelle Ausrichtung machte sie dennoch

$18 \quad$ Kaiser (1958).

19 Sisto (2006) 35-58. An Michele Sisto, dem Autor von grundlegenden Studien zur Rezeption der deutschen Literatur in Italien, geht mein Dank für die freundliche und großzügige Unterstützung. Ohne seine Hilfe wäre diese Forschungsarbeit infolge der derzeitigen Unzugänglichkeit der Verlagsarchive von Feltrinelli erheblich erschwert worden.

20 Ferretti (2004).

21 Cases (2013).

22 Magris (2009). 
unempfänglich für das Verständnis und die Wertschätzung der Provokation des jungen Grass. Verschafft man sich einen Überblick über die zahlreichen Ansichten der damaligen Verlagshäuser, so gelangt man zu dem weitverbreiteten Eindruck, der Roman habe die Mehrheit der Redakteure und Berater, die dessen Botschaft für befremdlich oder schwer zu entziffern hielten, insofern verwirrt, als diese den durch den Autor verkörperten Generationsbruch noch verschärfe. Lavinia Mazzucchettis Urteil ist in diesem Zusammenhang wohl der deutlichste Beitrag, der dem Mailänder Verlag eine Lesart in drastischem Stil nahelegte

Leider habe ich (wer wird uns je dafür entschädigen, wenn unser Gehirn nach so vieler derartiger uns aufgezwungener Lektüre Schaden nimmt ... ?) nicht alles, wohl aber einen Großteil dieses preisgekrönten Meisterwerkes des jungen Debutanten gelesen. Mir scheint, dass ich nichts aufs Spiel setze, wenn ich Mondadori von jeglicher Absicht des Erwerbs abrate. Sollte das Buch, dessen Intelligenz ich abstreite, in Zukunft dennoch groß heraus kommen, umso schlimmer. Es wird nie ein Kassenschlager werden. Es ist langweilig, ekelerregend superdeutsch, barock, überflüssig[...] Natürlich lässt das Buch auf eine gewisse Begabung schließen, eine besondere Gabe, Seiten hervorzubringen, so wie Wasser aus dem Wasserhahn läuft, um durch abgekartete Selbstanalysen und Symbolkraft die ganze Kloake jeweils mit dem entsprechenden Unterbewusstsein zu verwässern. Aber nun jagt ihn zum Teufel und lasst uns nicht verzweifeln, wenn ein anderer Verleger mit weniger prähistorichen Beratern als ich es bin, anbeißt und die fast 700 Seiten dieses Romans übersetzen lässt und und druckt. ${ }^{23}$

Auch das Urteil des Autors Elio Vittorini, eines weiteren einflussreichen Unternehmensberaters von Mondadori, war voller Bedenken und befürwortete ohne lange zu überlegen den Verriss: „Ein nur halb gelungener Versuch[...] Auf jeden Fall ein überzogenes Gemisch. Deshalb können wir das Buch ruhig von unserer Liste streichen“. ${ }^{24}$

Erst später wird er, infolge seines Beitrags zur internationalen Formentor Preisverleihung 1962 - obwohl er dem etwas schwierigeren Uwe Johnson den

23 Sisto (2009) 17. Sisto unterstreicht auch eine andere von Bruno Maffi für Mondadori geäußerte Meinung, der sich nicht imstande fühlt den verborgenen Sinn hinter der „eher mechanischen und gemeinen als tiefgründigen Phantasie“ des Protagonisten von Grass' Roman zu verstehen.

24 Sisto (2009), 18. Hervorhebung Calvinos. 
Vorzug gibt - Grass mit seiner Fabulierlust als eine Art Chagall der Literatur schätzen lernen, und zwar „einen Chagall, der sowohl aus der Geschichte als auch der Politik die Elemente entnimmt, aus denen sich seine Fabel zusammensetzt." ${ }^{\text {25 }}$

Bei Einaudi - einem Verlag, der bereits vor allem in seinen Essayreihen etwas sensibler auf die neuen Kulturfermente reagiert - brachte auch Cesare Cases mit Verdruss eine ablehnende Haltung zum Ausdruck, die mit der Zeit zu einer zur Schau gestellten Idiosynkrasie gegenüber Grass wurde. Dieser Autor sei seiner Meinung nach ein Anzeichen für eine der unseligen Tendenzen in der zeitgenössischen Literatur, und zwar „unnütz und unlesbar zu sein, jedoch fähig viel Sand in die Augen zu streuen“. ${ }^{26}$ Offensichtlich ist das, was bei der Blechtrommel auf Ablehnung stößt, gerade ihre unabänderliche Rätselhaftigkeit und Zweideutigkeit, was indessen für die Neuavantgardisten eben das Wesen der Literatur an sich ausmacht: „Ein literarischer Text ist - so behauptet beispielsweise Edoardo Sanguineti - ein Text, der sich in Form eines Enigmas darstellt. Wo Poesie ist, da ist immer auch ein Rätsel““. ${ }^{27}$

Die Wende für Grass' Roman erfolgte, als in diesem eher konservativen Szenarium, das im Grunde nicht auf angemessene Art und Weise auf die neuen Herausforderungen des kulturellen Marktes zu reagieren vermochte, der junge Verleger Giangiacomo Feltrinelli (1926-1972) auftauchte, der 1955 in Mailand das gleichnamige Verlagshaus gegründet hatte. ${ }^{28}$ Als Sohn einer sehr wohlhabenden Unternehmerfamilie hatte er eine ,zornige' Jugend hinter sich, in der er gegen den Strom geschwommen war: mit unregelmäßigem und abgebrochenem Studium trat er aktiv der PCI bei und war im Verlagswesen der Partei tätig, aus der er 1956 austrat. Mit seinem dank des Familienvermögens auf finanzieller Ebene relativ selbstständigen Verlagshaus wollte er eine offene und vorurteilsfreie Richtung verfolgen, unter Verbindung von unternehmerischem Elan, politischem Aktivismus im Zeichen des Antifaschismus und Marxismus und der Suche nach in jeder Hinsicht Neuem. Sein Einsatz bestand darin, Italien vom Provinzialismus zu befreien und die von den zwanzig Jahren der Diktatur hinterlassenen kulturellen Lücken wieder aufzufüllen. „Der Stand der Kultur unseres Landes - so steht es im Programm des Verlagshauses für

25 Vitttorini (1962) 4-6.

26 Cases (2013), LIII. Die Position von Cases verhinderte dennoch nicht, dass Grass Anfang der 7oer Jahre Feltrinelli verließ und alle seine Werke bis heute bei Einaudi publiziert hat. Dieser Abschnitt verweist deutlich darauf, dass Grass das Umfeld der experimentellen Avantgarde verlässt und seinen Einzug unter die Klassiker der Gegenwartsliteratur begeht.

27 Sanguineti (1987) 210.

28 Cesana (2010). 
die Jahre $1955^{-1965}$ - wird auch und in großem Ausmaß davon abhängen, was die Italiener im Bereich der Gebrauchsliteratur gelesen haben werden. ${ }^{29}$ Mit der Entdeckung und Veröffentlichung zweier unbekannter und umstrittener Meisterwerke konnte sich der neue Verlag sensationell vor der öffentlichen Meinung behaupten: Boris Pasternaks Doktor Schiwago von 1957 und 1958 Giuseppe Tomasi di Lampedusas Der Leopard. Im Jahr 1958 besiegelte Feltrinellis Eheschließung mit der deutschen Fotografin Inge Schöntal ein privilegiertes Verhältnis zu Deutschland. Dabei war sein erklärtes Vorbild der geniale Kurt Wolf, „der finanziell viele Verlagshäuser ruiniert hat, wobei er kulturell immer Recht behalten sollte“. ${ }^{30}$

Die moderne deutsche Literatur sollte später bei seinen verlegerischen Entscheidungen eine erstrangige Position einnehmen, als 1960 der 28-Jährige Enrico Fillippini (1932-1988) Redakteur für die ausländische Literatur wurde. ${ }^{31}$ In der Schweiz geboren und in Mailand mit einem Philosophiestudium zum eigenständigen Schriftsteller ausgebildet, frequentiert er den Kreis der Neuavantgardisten und wird später zu einem der Mitbegründer der Gruppe 63.

Er nimmt an einigen Versammlungen der Gruppe 47 teil, mit einem Augenmerk auf all dem Neuen, was sich in der Kulturszene jenseits der Alpen abspielt, insbesondere auf die Autoren, die wie er der jungen Generation der ,Skeptiker' (Helmut Schelsky) der Jahrgänge Ende der 2oer Jahre angehören. Diese Generation wird zum Wortführer für ein politisches und ästhetisches Missbehagen gegenüber den herrschenden Verhältnissen. Über Filippini entscheidet sich der Verlag Feltrinelli mit Entschlossenheit und ungeachtet aller Risiken für die Publikation von damals in Italien völlig unbekannten Vertretern der nonkonformistischen Literatur wie Uwe Johnson, Hans Magnus Enzensberger, Max Frisch, Friedrich Dürrenmatt, Ingeborg Bachmann und Rolf Hochhuth. Das Schaustück aus dieser Reihe war 1962 eine Anthologie mit dem programmatischen Titel Il dissenso: 19 nuovi scrittori tedeschi ["Der Dissens: 19 neue deutsche Schriftsteller"], mit Hans Bender als Herausgeber. Außerdem übersetzte Filippini selbst mit brennender Leidenschaft die ausgewählten Texte und infolge der Dringlichkeit der Veröffentlichung nicht selten ohne eine gewisse Stringenz im Ausdruck in der italienischen Version.

Grass fand vollkommenen Eingang in die Interessenssphäre dieses neugierigen Redakteurs. Ihn verband eine ganz besondere Freundschaft und harmonische Übereinstimmung mit dem Lübecker Schriftsteller. Damals hatte das Verlagshaus Bompiani, wie bereits erwähnt, die Rechte für Die

\footnotetext{
29 Feltrinelli (1965), XI.

$30 \quad$ Feltrinelli (1999) 117-145.

$31 \quad$ Sisto (2009).
} 
Blechtrommel bereits erworben. Aber Filippini ließ sich ein Jahr später Katz und Maus nicht entgehen, wovon er selbst die italienische Version lieferte. Als Bompiani somit nach einigen Monaten Feltrinelli die Möglichkeit bot, das ,aufsehenerregende` Werk Tamburo di latta zu übernehmen, nahm Filippini begeistert die Gelegenheit wahr, dem Publikum selbstverständlich zuerst den Roman und 1964 die Novelle Gatto e topo [Katz und Maus], den zweiten Teil der Danziger Trilogie, vorzustellen. ${ }^{32}$

Der Vermarktung des Romans ging eine wohldurchdachte Werbekampagne voraus. Der Zeitschrift Il Verri - dem, wie bereits erwähnt, maßgeblichsten Presseorgan der Neuavantgardisten - wurde der Vorabdruck des Kapitels Glaube, Hoffnung, Liebe anvertraut, eine Entscheidung, die unmittelbar auf das Thema setzte, das, aktueller denn je, auch in Italien eine Debatte über die unbewältigte Vergangenheit ausgelöst hatte. Die Einleitung des Textes enthielt eine kritische Anmerkung des Germanisten Giuliano Baioni, welcher trotz einiger Vorbehalte aufgrund des exzessiven Antikonformismus von Grass, Il tamburo di latta als eine neue Etappe in der Geschichte des Entwicklungsromans beschrieb:

Aber sein Held, der ein unverwechselbares Einzelwesen sein will und gleichzeitig auch ein idealer Vertreter seiner Zeit, kann nur der unvoreingenommene Chronist einer deformierten und monströsen Epoche sein, insofern er sich selbst als eine missgestaltete und monströse Kreatur erweist. [...] Deutschland, das bis heute noch nicht sein Drama aufzuschreiben vermocht hat, liefert uns davon momentan nur eine Parodie, und zwar eine Parodie um die schrecklichste aller Wahrheiten über sämtliche Werte und Erscheinungsformen der deutschen Gesellschaft auszusprechen. ${ }^{33}$

Der Roman kam schließlich in der namhaften Reihe I narratori heraus, dort, wo auch die großen Namen der zeitgenössischen Literatur erscheinen, wie Lawrence Durrel, Malcom Lowry, Yukio Mishima, Henry Miller, und unter den italienischen Autoren Arbasino mit Fratellid'Italia (1960), einem vorher bereits bei Feltrinelli selbst infolge von Giorgio Bassanis Beurteilung, durchgefallenen Roman. Dabei handelt es sich, wie man sehen wird, meistens um Schriftsteller, die mit Grass eine umstrittene und oft anstoßerregende Rezension teilen.

$3^{2}$ Der dritte Teil Hundejahre (Anni di cani) erschien in derselben Reihe im Jahr 1966. Filippini zeichnete ebenfalls die Übersetzung. 
Il tamburo di latta wurde als ein mit einem reichen Peritext (Schutzumschlag, Bauchbinde) ausgestatteter kartonierter Band präsentiert. Dies geschah aufgrund der Lancierung eines unbekannten Autors, an den Feltrinellis Publikum, bestehend aus neugierigen und antikonformistischen Lesern, gewisse Erwartungen stellte. Auf dem eleganten roten Einband befand sich oben nur die eigenhändige Unterschrift von Günter Grass; auf dem Schutzumschlag auf dem Frontispiz war, in idealer Abstimmung von Autor und Text, unter dem Titel ein Schwarzweißfoto des Schriftstellers abgebildet. Mit angezündeter Zigarette im Mund, einer bürgerlichen Krawatte, die ironisch mit dem eher zerknitterten Hemd kontrastierte, und mit einem vagen, scheinbar gleichgültigen Lächeln auf den von dem damals bereits berühmten Schnurrbart eingerahmten Lippen, war Grass im Begriff, für die Fans der italienischen Neuavantgarde zu einer perfekten ausländischen Ikone zu werden. ${ }^{34}$ Der hintere Klappentext enthielt dagegen ein umfangreiches Zitat aus der Rezension, die Hans Magnus Enzensberger 1959 in den Frankfurter Heften der Blechtrommel gewidmet hatte. Eine wegweisende und nahezu programmatische Entscheidung, denn sie verband die beiden radikalsten Exponenten der neuen deutschen Literatur miteinander und verwies zugleich auf deren Einklang mit dem ikonoklastischen Programm der experimentellen Richtung in Italien:

Die Blechtrommel kennt keine Tabus. Gewalttätig wirkt dieser Roman, weil er alles berührt, als wäre es antastbar. [...] Immer wieder tritt die Erzählung in jene verbotene Sphäre ein, wo sich Ekel und Sexualität, Tod und Blasphemie begegnen [...] Grass jagt nicht, wie Henry Miller, hinter dem Tabu her: er bemerkt es einfach nicht $[\ldots]{ }^{35}$

In Enzensbergers Worten verlagerte sich also der Akzent auf den blasphemischen und erotischen Charakter des Werkes, der auch durch die Bezugnahme auf Miller betont wurde, von dem Feltrinelli gerade im selben Jahr heimlich Il Tropico del cancro veröffentlicht hatte. Der Verleger beanspruchte und zelebrierte somit auch seine eigene Souveränität gegenüber religiösen oder moralischen Beeinflussungen unterschiedlicher Herkunft. Schließlich zielte die dieser Erstausgabe hinzugefügte Bauchbinde mit ihren sich wiederholenden Superlativen auf eine noch sensationellere und skandalösere Wirkung ab. Sie lautete wie folgt:

34 Interessant ist die Feststellung, dass auch Vertreter der italienischen Avantgarde ähnlich fotografisch portraitiert wurden. Zum Beispiel Edoardo Sanguineti mit Krawatte und stets mit einer Zigarette im Mund. 
Der Aufsehen erregendste Roman der jungen deutschen Literatur, ein absoluter Bestseller der Nachkriegszeit in Deutschland, der meistverkaufte ausländische Roman in Frankreich. Il tamburo di latta von Günter Grass. Der Nationalsozialismus aus der Perspektive eines erotomanen Zwerges.

Abgesehen von dieser perfekten Verpackung wurde das Erscheinen des Romans auch seitens einer mit der Verbreitung einhergehenden, weisen Verlagsregie unterstützt. Grass konnte sich als Persönlichkeit der Literaturszene dank zahlreicher Interviews in den bedeutenden Feuilletons und in den Massenmedien behaupten. Der Roman erzielte somit ungeachtet seiner proklamierten Komplexität einen großen Erfolg: die erste Taschenbuchausgabe, in der damals als Übersetzer nur der Name von Lia Secci angeführt wurde, erschien 1965 (Reihe,Gli Astri'); worauf 1974 eine noch populärere Ausgabe (in der Reihe ,Universale Economica') folgte, die, wie die vorhergehende, auf der Umschlagseite eine von Grass persönlich unterzeichnete Radierung zeigte, da er inzwischen der Öffentlichkeit auch als Künstler bekannt war.

Dieser glückliche Ausgang war eine Bestätigung dessen, was nicht selten bei umstrittenen Publikationen geschieht: oftmals zieht der Autor einen großen Nutzen daraus und wird vollends entschädigt, da er schließlich beim erbittertsten, bzw. motiviertesten Verleger in Hinblick auf sein Werk landet, der in der Lage ist, es in den für eine erfolgreiche Rezeption geeignetsten Umlauf zu bringen. ${ }^{36}$

Dies wird auch in der Reaktion der Literaturkritk auf Grass' Roman bestätigt. Die Presse unterschiedlichster Couleur beschäftigte sich noch jahrelang nach seinem Erscheinen mit Il Tamburo di latta, sodass sich die vom Buch ausgelöste breite Debatte heute in ihren wesentlichen Zügen nachvollziehen lässt. Angesichts des Umfangs und der vom Roman repräsentierten Neuheit brauchten die italienischen Literaturkritiker dennoch Zeit zur Erstellung einer Interpretation des Textes, die die Hintergründe erläutern konnte, wie Luigi Baldacci, einer der scharfsinnigsten Rezensenten dieser Erstausgabe, mit viel Verstand bemerkte:

Wir haben es mit einem Werk zu tun, das gewissermaßen mehr oder weniger erst in einer nahen Zukunft ganz gelesen werden kann: ein Buch, über dessen Inhalt man Bericht erstatten kann und muss, wobei man vor sich selbst und anderen nicht verbergen sollte, dass viele seiner Aussagen

$36 \quad$ Ferretti (2004) 3-4. 
nur zur Bestätigung dessen einleuchtend erscheinen, was die Gattung ,Roman' von nun an bis in vielen Jahren sein wird. ${ }^{37}$

Die ersten Reaktionen waren durchaus recht oberflächlich und unwissend bezüglich des historischen Kontextes, der Oskars Zorn auslöst. Nur wenige Tage nach der Erscheinung im Buchhandel beschäftigte sich Paolo Pavolini in der Turiner Tageszeitung La Stampa mit Grass im Rahmen einer umfassenden Analyse des ,Wiedererwachens der Kultur in Deutschland'. Darin kam der Danziger Schriftsteller neben Uwe Johnson und Erich Kuby als Vertreter eines verbreiteten Kulturpessimismus vor, der seinerseits in einem ironischen und entmystifizierenden politischen Indifferentismus seinen Ausdruck finde:

Wenn ihr heute in Deutschland in Frieden leben wollt - das wäre die Lehre des barocken, exzessiven Romans - dann haltet euch vom Erwachsenwerden fern, lasst euch für verrückt erklären, beschäftigt euch mit nichtigen und lauten Dingen, liebt euch in der Familie und missachtet die Welt um euch herum. ${ }^{38}$

Das, was für Pavolini eine groteske, aber geniale Absage an jegliches Engagement in der Gegenwart war, erscheint Pietro Citati eine Woche später als übertriebene Überschwänglichkeit, hinter deren „spöttischem und respektlosem Blick“ sich nur ein vulgärer und törichter Nihilismus verberge. Die „wahre Muse“ des Romans sei das „Märchen: die sensationelle und überladene Erfindung, die die Dinge ständig mit einer Farbschicht ummantele“. ${ }^{39}$ Der damals bereits einflussreiche, junge italienische Kritiker scheint sich somit bei seiner Argumentation auf das nunmehr berühmte Urteil von Reich-Ranicki anlässlich des Erscheinens der Blechtrommel in der BRD zu berufen. ${ }^{40}$

Nach einigen Monaten verhärten sich um Grass und seinen Text zwei gegensätzliche Positionen der Literaturkritik, die größtenteils die bereits erwähnten kulturellen Formierungen des Landes widerspiegeln. Die konservativsten Rezensenten richten ihre Offensive gegen einen anmaßenden und künstlerisch gescheiterten Roman, dessen übertriebener Erfolg lediglich literarischen Modeerscheinungen und einfach dem Geschmack am Skandal zu verdanken sei. Aber - so zum Beispiel das moralistische Urteil des Journalisten Ernesto

37 Baldacci (1963).

38 Pavolini (1962).

39 Citati (1962).

40 „Nicht selten ist das Feuer dieser Prosa echt. Aber von dem Wasser ist allzu viel da - und wir haben es nicht immer mit sauberem Wasser zu tun." Reich-Ranicki (1960). 
Travi - ,jede Profanierung, selbst die schlimmste, selbst die absoluteste, endet schließlich notgedrungen mit dem Eingeständnis, dass man nicht ständig mit einem so bitteren Spiel leben kann [...] man kann nicht alles und alle unaufhörlich verspotten“. ${ }^{41}$

In dem drastischen Verriss wird das Buch von Grass mit den Werken der ,verflixten' beat generation in Verbindung gebracht, oder mit Salingers jüngst erschienenen Werk The Catcher in the Rye und dessen ,pseudopopulärer Idiotie؛. Der Danziger Schriftsteller wird zum Prototypen „der schrecklichen und pausbackigen Avantgarde dieser demokratischen Zeiten, in denen es reicht, die Institutionen, die Mütter, die Großmütter und die alten Tanten mit Füßen zu treten um Erfolg zu haben".42 Auch Giorgio Zampa weicht im Corriere della Sera, der wichtigsten bürgerlich-liberalen Tageszeitung Italiens, nicht sehr von den strengen Beurteilungen ab, die die moderate Kritik für Grass auch als Vertreter eines verachteten und gefürchteten literarischen Antikonformismus bereithält. Der Erfolg von Tamburo di latta ist für Zampa das Ergebnis der geschickten Werbestrategie „einer als Gruppe 47 getauften Art Bruderschaft: einige brillante Ideen, eine gute Portion moralischer Skrupellosigkeit, sprachliche Kunststücke, mehr nicht. Wenn seine Grausamkeit und Gewalt nur eine Pose sind, dann bleibt nur noch eine erbärmliche Traurigkeit, eine unbestimmbare Sehnsucht, die sich - und das ist das letzte Wort des Rezensenten - in kaum wahrnehmbarem Gestammel ausdrückt". 43

Von dieser Richtung hebt sich sehr bald eine gut vorbereitete Gruppe aufgeschlossenerer Rezensenten ab, die eine tiefgreifende Analyse und Kontextualisierung des Romans in der deutschen Nachkriegszeit vornehmen, wobei sie auch über die Ursachen der Vorbehalte gegenüber Grass in Italien nachdenken. Cesare Giardini bemerkte beispielsweise in einem in vielen Zeitungen erschienenen Artikel, es sei unvermeidlich, dass in einem Land, „wo die Literatur, von ein paar Ausnahmen abgesehen, von langweiligen und verhaltenen, rein verbalen Provokationen lebt“, jene außerodentliche und hemmungslose Erzählung mit einem gewissen Misstrauen aufgenommen werde. $^{44}$

Liest man heute die zahlreichen Rezensionen noch einmal, so ist vor allem eine interessante Gegenüberstellung zwischen zwei unterschiedlichen Lesarten der Beziehung zwischen Oskar und der deutschen Geschichte festzustellen, Interpretationen, die bis heute in der Rezeptionsgeschichte der

\footnotetext{
41 Travi (1960).

42 Orsera (1963).

43 Zampa (1963).

44 Giardini (1963).
} 
Blechtrommel aufrecht erhalten wurden..$^{45}$ Auf der einen Seite schlägt man vor, wie beispielsweise Michele Rago in der Unità, den Roman als die erste vollständige Aufarbeitung des Nationalsozialismus in der Erzählliteratur anzusehen und einen geschichtspolitischen Interpretationsansatz ins Leben zu rufen, der in Italien bis zur Annäherung des Werks von Grass an Thomas Manns Doktor Faustus übernommen wurde. ${ }^{46}$ Als ein zugleich komisches wie bitteres Fresko entlarve Il tamburo di latta „politische Konstrukte, fanatische oder pseudoreligiöse Heuchelei, erotische Phantasien, krankhaftes sexuelles Verlangen [...] mit einer Naivität, die einen erschaudern lässt" ${ }^{4}{ }^{47}$ Durch den unmoralischen und entmystifizierenden Ton seines Zwerges mache Grass sogar einen ersten Schritt in Richtung der Befreiung Deutschlands von seinem ihm in der Nachkriegszeit auferlegten Schuldgefühl, und Oskar entledige sich mit seinem Zynismus der "ganzen schweren Rhetorik der Reue“.48 In diesem Sinne vergleicht auch Carla Marzi Grass' Satire mit der von George Grosz und Otto Dix, wobei sie zu Recht auch den Generationsaspekt hervorhebt:

Nach einer großen Tragödie wenden sich die jüngeren und sensibleren Schriftsteller, diejenigen, die angesichts des allgemeinen Wahnsinns des sie überrollenden Krieges, der vor ihren Augen alle Mythen und die alten Wertvorstellungen mit sich hinwegriss, entwaffnet zusahen, mit Abscheu der Vergangenheit zu und rühren in deren Asche um sicher zu gehen, dass nicht einmal ein Funke mehr glimmt. ${ }^{49}$

Auf der Gegenseite ist man indessen geneigt, Oskars Geschichte einer jeglichen politischen Bedeutung zu entledigen und seine Art des Protestes als eine ganz und gar nihilistische Absage an die Geschichte und die Gesellschaft auszulegen. Der Trommler sei vielmehr eine existentielle und tragische Allegorie auf die Situation des Menschen, „etwas, das über die Geschichte hinausgeht, was ihr von allen Seiten entgleitet, was die Geschichte nicht mehr beinhaltet" ${ }^{\text {" }} 0$ Der Rahmen Deutschlands, des Nationalsozialismus und der Nachkriegszeit seien daher nicht im einfachen referentiellen Sinne zu verstehen und Grass' Held würde somit zum Symbol einer um all ihrer Werte beraubten Menschheit, „und er tendiert zu zwei vom Kern der Vernunft gleichermaßen entfernten

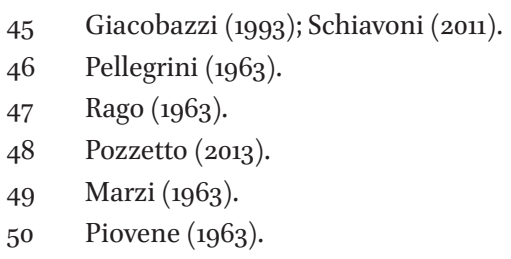


Richtungen: der Religion und der Sexualität“. ${ }^{51}$ In dieser Hinsicht nahm Il tamburo di latta an dem in Italien selbst stattfindenden, bereits erwähnten Kampf gegen den (Neo-)Realismus teil. Der Germanist Giorgio Cusatelli bereichert seine eingehende Analyse des Texte um die Kategorie des Barock. Dabei betont er, dass dieser das Ausdrucksmittel einer Kultur sei, die dem Versuch erlegen sei, der Realität eine vernunftsmäßige Ordnung aufzuerlegen. Der Antirealismus von Grass wird zur erbitterten Suche nach dem Extremen und Symbolhaften „als Sinngebung einer konkreten Gegebenheit, die diese in eine andere Dimension überträgt“ ${ }^{\star}{ }^{52}$

Es war nunmehr an der Zeit, dass auch Grass' Stil endlich nicht mehr als unerhörter Skandal auf Ablehnung stieß und man ihn sogar mit dem eines angesehenen Schriftstellers wie Carlo Emilio Gadda in Verbindung brachte. In der Wochenzeitschrift La fiera letteraria würdigt Gilda Musa seine neuexpressionistische sprachliche Virtuosität, die dazu diene, die finstersten Winkel der menschlichen Ängste auszuloten, wobei sich "literarische Ausdrücke, Dialekte, Argot allmählich und je nach improvisierten Regeln in bunten Variationen miteinander vermischen“. Außerdem entgeht Musa auch nicht ein bis jetzt mit Stillschweigen übergangener Aspekt des Romans, nämlich die (bekanntlich von Grass selbst immer wieder betonte) Absicht, die Erinnerung an eine verloren gegangene Welt durch die Dichtung zu wahren. Mit einer sinnvollen perspektivischen Erweiterung wird das Danzig von Grass mit Rezzoris Cernopol verglichen, oder auch mit dem Berlin von Uwe Johnson und dem Wasserburg von Martin Walser. ${ }^{53}$

Im August 1963 kommen schließlich die italienischen Neoavantgardisten mit Renato Barilli bezüglich des Tamburo di latta zu Wort. Es überrascht nicht, dass auch in diesem Fall weniger die Geschichte und die politische und ideologische Einstellung von Grass den Kritiker interessiert als vielmehr die von ihm angewandte Erzählstrategie, um die Rhetorik der Macht und wiederum die falsche Moral der ihn umgebenden kleinbürgerlichen Welt ins Lächerliche zu ziehen. Barilli beschreibt diese Strategie mit der genialen Formulierung ,transzendentales Zwergtum. Die Missbildungen, die sich Oskar absichtlich selbst zufügt, sind in dieser Hinsicht die „notwendigen Voraussetzungen zur Erlangung eines authentischen Wissens über die Welt“. ${ }^{4}$

Innerhalb kurzer Zeit scheint also der anfängliche Widerstand des Konservatismus, sowohl von rechts als auch von links, gegen das absolute Novum des

\footnotetext{
$51 \quad$ Baldacci (1963).

$5^{2}$ Cusatelli (1963).

53 Musa (1963).

54 Barilli (1963).
} 
Romans von Grass bezwungen. Dies ist das Ergebnis einer Art Revolution, die sich im Bereich der italienischen Literatur selbst ereignet hat, in Gang gesetzt durch das gemeinsame Agieren der innovativen Verlagspolitik von Feltrinelli und dem Engagement der von experimentellen Impulsen angetriebenen Literaturkritiker, die die Kulturszene dieser Zeit belebten.

Die Monate, in denen der Trommler von Danzig sich einen Platz in den italienischen Buchhandlungen erobert, sind auch der Zeitraum, in dem die Gruppe 63 den Fermenten der Neuavantgarde auch theoretisch Form verleiht. Überblickt man heute wieder die erste Rezeption zum Tamburo di latta und nimmt die darin enthaltene fortschreitende Öffnung zur Kenntnis, so trägt dies auch zum Verständnis eines Abschnitts der Geschichte in einem Land bei, das Anfang der 6oer Jahre durch eine Auseinandersetzung mit den aus dem Ausland kommenden Provokationen seine eigenen Bedürfnisse nach Modernisierung und nach einem Ausweg aus der Stagnation der frühen Nachkriegszeit unter Beweis stellte. Seitdem hat der Roman seinen Status als Klassiker der Moderne erworben und aufrechterhalten. Im Jahr 2009 kam auch der Verleger Feltrinelli nicht umhin, mit einer neuen Übersetzung von Bruna Bianchi den fünfzigsten Jahrestag von Grass' Buch zu feiern, was als eine der entscheidenden Etappen in der Geschichte dieses Mailänder Verlagshauses zu verstehen ist. Nach einem Weg mit Hindernissen hat der anarchische Trommler, der respektlose Schelm, welcher das durch den Wirtschaftsboom abgestumpfte bürgerliche Bewusstsein irritiert, aber auch aufgeweckt hat, ${ }^{55}$ sich auch in Italien mit seiner durchschlagenden Kraft einen bedeutenden Platz in der Vorstellungswelt unserer modernen Zeit gesichert.

\section{Literatur}

Albrecht C., (et. al.), Die intellektuelle Gründung der Bundesrepublik. Eine Wirkungsgeschichte der Frankfurter Schule (Frankfurt am Main, 2000).

Antonio, A., Maledizioni. Processi, sequestri, censure a scrittori e editori in Italia dal dopoguerra a oggi anzi a domani (Torino 2013).

Baldacci, L., „La diabolica storia del nano di Danzica“, in Epoca, 24. Februar 1963 (and in Il giornale del mattino, April 9, 1963).

55 „Für die Deutschen“, so steht in einer Rezension zur Neuausgabe von 2009, „war es, als hätte plötzlich jemand eine Wand eingeschlagen und als wäre die Luft in ein viel zu lange verschlossenes Zimmer eingetreten“. Vanna Vannuccini, „Il libro che cambiò la Germania“, La Repubblica, 7.1.2009. 
Banchelli, E., „Nonkonformismus und Experiment. Einleitung“, in Agazzi, E./E. Schütz (Hg.), Handbuch Nachkriegskultur. Literatur, Sachbuch und Film in Deutschland (19451962), (Berlin/Boston, 2013), 553-561.

Barilli, R., „Uomini e nani. Un triangolo autentico“, in Il Mulino, August 1963.

Barilli, R./A. Guglielmi (Hg.), Gruppo 63 : critica e teoria (Milano, 1976).

Berbig R./H. Markus (Hg.), Berliner Hefte zur Geschichte des literarischen Lebens, 8, 2008 [„Das literarische Jahr 1959”].

Calvino, I., „Lettera di dimissioni dal PCI“, in L'Unità, 7. August 1957.

Cases, C., Scegliendo e scartando. Pareri di lettura (Torino, 2013).

Cesana, R., ,Libri necessari'. Le edizioni Feltrinelli 1955-1965 (Milano, 2010).

Citati, P., „Protesta contro tutto picchiando sul tamburo di latta“, in Il Giorno, 28. November 1962.

Di Stefano, P., „Il tamburo censurato. Riemerge l'edizione fantasma del capolavoro buttato al macero", in Corriere della sera, 8. February 2007.

Cusatelli, G., „Un moralista barocco“, in Questo e altro, 3 (1963).

Enzensberger, H.M., „Wilhelm Meister, auf Blech getrommelt“, in Einzelheiten (Frankfurt am Main, 1962), 223.

Feltrinelli 1955-1965. Guida alla lettura e catalogo generale delle edizioni Feltrinelli (Milano, 1965).

Feltrinelli, C., Senior service (Milano, 1999).

Ferretti, G., Storia dell'editoria letteraria in Italia 1945-2003 (Torino, 2004).

Ferretti, G., Siamo spiacenti. Controstoria dell'editoria italiana attraverso irifiuti editoriali (Milano, 2012), 117-118.

Filippini, E., „Dalla parte del nano“, in La Repubblica, 3. Dezember 1978 (in: E. Filippini, La verità del gatto. Interviste e ritratti 1977-1987 (Torino, 1990), 34).

Giacobazzi, C., La storia risvegliata. Il barocco nella 'Trilogia di Danzica' di Günter Grass: le forme, le funzioni, gli esiti (Bologna, 1993).

Giardini, C., „Il nano col tamburo“, in Il Piccolo, 4. März 1963.

Guglielmi, A., „L'officina di Gadda“, in La fiera letteraria, May 25, 1958 (in: Barilli (1976), pp. 137-142).

Guglielmi, A., „La poetica della svalutazione“, in Palatina, September 1959 (in Barilli (1976), 152).

Guglielmi, A., „Pasolini, maestro di vita“, in Il Verri, 3, 1960. (in Barilli (1976), 169-174).

Häntzschel, G./S. Hanuschek/U. Leuschner (Hg.), Treibhaus. Jahrbuch für die Literatur der fünfziger Jahre , 5, 2009 [„Das Jahr 1959 in der deutschsprachigen Literatur“].

Kaiser, J. „Die Gruppe 47 lebt auf. Zur Herbsttagung in Großholzleute“, in Frankfurter Allgemeine Zeitung, 5. November $195^{8}$.

Magris, C., „Cesare Cases. Commemorazione“, in Atti dell'Accademia delle Scienze di Torino. Classe di Scienze Morali, Storiche e Filologiche, vol. 143 (Torino, 2009), 30.

Marzi, C., „Un tamburo per svegliare la Germania“, in Paese sera, 29. März 1963. 
Milano, P., „Il gusto feroce di raccontare“, in L'Espresso, 10. Juli 1960.

Musa, G., „L'assoluta fantasia di Günter Grass“, in La fiera letteraria, 27. Januar 1963.

Orsera, G. „Dove finiscono gli ideali“, in Il Borghese, 31. Januar 1963.

Pavolini, P., „C'è un risveglio della cultura in Germania ma gli intellettuali incontrano poca fortuna“, in La Stampa, 20. November 1962.

Pellegrini, A., „Arriva Grass con odore di bruciaticcio“, in Gazzetta del popolo, 6. Februar 1963.

Piovene, G., „Il tedesco Grass col 'Tamburo di latta' ci dà la favola della totale disperazione“, in La Stampa, 26. März 1963.

Pozzetto, A., „Il tamburo di latta“, in L'Italia che scrive, April 2013.

Rago, M., „Un Tamburo di latta contro Hitler“, in L’Unità, 23. Januar 1963.

Reich-Ranicki, M., „Auf gut Glűck getrommelt“, in Die Zeit, 1. Januar 1960.

Sanguineti, E., La missione del critico (Torino, 1987).

Schiavoni, G., Günter Grass, Un tedesco contro l'oblio (Roma, 2011).

Sisto, M., „I tedeschi di Feltrinelli': die deutsche Literatur der 6oer Jahre in Italien“, in Jahrbuch für Internationale Germanistik, 38/1 (2006), 35-58.

Sisto, M., „Enrico Filippini, sintesi di movimento“, in Nazione indiana, 2. September 2009 $<$ http://www.nazioneindiana.com/2009/10/28/enrico-filippini-sintesi-di-movi mento/>.

Sorge, P., „Un'esplosione di rabbia contro i mostri tedeschi“, in La Repubblica, 4. Februar 2003.

Travi, E., „L'inviso e malefico nano batte un tamburo di latta“, in L'Italia, 5. Januar 1963. Vittorini, E., „Comunicazione a Formentor“, in Il Menabò, 5 (1962), 4-6.

Zampa, G., „Un tamburo di latta per uno scrittore-clown“, in Il Corriere della sera, 13. Februar 1963. 